Horizons philosophiques

\title{
Les objets de la sémiologie théâtrale : le texte et le spectacle
}

\section{Louise Vigeant}

Volume 1, numéro 1, automne 1990

Sémiotiques 1 : mises au point, mises en question

URI : https://id.erudit.org/iderudit/800861ar

DOI : https://doi.org/10.7202/800861ar

Aller au sommaire du numéro

Éditeur(s)

Collège Édouard-Montpetit

ISSN

1181-9227 (imprimé)

1920-2954 (numérique)

Découvrir la revue

Citer cet article

Vigeant, L. (1990). Les objets de la sémiologie théâtrale : le texte et le spectacle. Horizons philosophiques, 1(1), 57-79. https://doi.org/10.7202/800861ar d'utilisation que vous pouvez consulter en ligne.

https://apropos.erudit.org/fr/usagers/politique-dutilisation/ 


\section{Les objets de la sémiologie théâtrale : le texte et le spectacle}

La sémiologie se distingue des autres métalangages portant sur le théâtre - histoire, dramaturgie, critique, psychologie de l'acteur, etc. - surtout par son souci de formalisation. En effet, elle "aborde l'activité spectaculaire construite à partir de systèmes de signes organisés en ensembles signifiants d'une certaine manière. Elle englobe à la fois la production, la réception et les modèles du spectacle ${ }^{1}$ ». La sémiologie va donc identifier les éléments qui composent le théâtre - qu'elle considère comme des signes; elle se propose également de découvrir les principes de son organisation et de dévoiler les modes de fonctionnement de la signification dans cette forme d'art. La citation lance trois mots clés : production, réception et modèles du spectacle qui présentent les niveaux de recherche de la sémiologie théâtrale.

\section{L'objet de la sémiologie théâtrale}

Aussitôt qu'elle s'est intéressée au théâtre, la sémiologie a été confrontée au problème de la détermination de

1. A. Helbo, J.D. Johansen, P. Pavis, A. Ubersfeld, (sous la direction de), Théâtre/Modes d'approche, Bruxelles, Éditions Labor et Méridiens Klincksieck, 1987, p. 13. 
son objet. Car s'il est facile de voir que le code de la langue est l'objet de la linguistique - le modèle scientifique de la sémiologie -, ce n'est pas aussi simple quand on considère d'autres systèmes de signification et de communication, en particulier les textes artistiques, doublement modélisés (cf. Lotman).

II n'y a pas au théâtre, comme dans la langue naturelle, un signe minimal dont il s'agirait d'examiner les modes d'articulation (car comment déterminer un signe minimal quand la substance de l'expression est hétérogène?). On ne peut donc pas parler d'une langue théâtrale. Mais cela ne veut pas dire, pour autant, qu'on ne peut pas parler de codification au théâtre!

Alors, si l'objet de la sémiologie n'est pas une langue, est-ce que cela veut dire que l'on étudie le théâtre en tant que manifestation discursive? Si oui, on abandonne le modèle linguistique puisque, selon Saussure, la linguistique s'occupe de la langue et non de la parole, c'est-à-dire du discours singulier. Mais tous les problèmes ne sont pas réglés pour autant. En outre, quand on parle de theâtre, parle-t-on du texte dramatique ou du spectacle? Car il faut faire remarquer une singularité de l'événement théâtral qui ne manque pas de compliquer l'approche sémiologique : ses trois niveaux de communication. En effet, au théâtre, tour à tour, l'auteur, le metteur en scène et les comédiens sont des émetteurs qui du texte dramatique, qui de la mise en scène et qui, finalement, du spectacle (cf. Purkhardt). La question du passage du texte à la scène, de l'ordre de l'interprétance discursive, est d'ailleurs apparue assez tôt comme l'un des sujets de prédilection de la sémiologie théâtrale. Mais celle-ci a résisté longtemps à traiter du spectacle, confrontée qu'elle était à l'absence même d'objet puisque, par définition, la représentation théâtrale est éphémère et ne survit que dans la mémoire des spectateurs.

Dans cet effort d'attribuer un objet à la sémiologie, Évelyne Ertel distinguait, en 1977, deux sémiologies : l'une 
du code, l'autre du message, selon qu'on s'attache à dégager une quelconque "langue» théâtrale, justement, ou qu'on analyse une articulation spécifique de cette "langue" (une parole, en termes saussuriens) :

La première (la sémiologie des codes) travaillant sur un corpus déterminé (un ensemble de mises en scène différentes regroupées avec ou sans principe; le principe peut être le genre, la pièce, l'auteur, l'époque, etc.), s'efforcera de dégager et de décrire les codes théâtraux communs à tous ces spectacles. La seconde, travaillant sur une mise en scène particulière, s'efforcera d'analyser le plus grand nombre de codes à l'œuvre, qu'ils soient théâtraux ou non, et la manière singulière dont ils sont structurés (le système textuel particulier de cette représentation) ${ }^{2}$.

En d'autres mots, si la deuxième sémiologie s'intéresse aux discours, la première, elle, chercherait les "structures profondes" (telles que les a découvertes Greimas pour le récit) qui permettraient de parler d'une langue théâtrale "présupposée par toute manifestation discursive et qui, en même temps, prédétermine les conditions de la "mise en discours" (c'est-à-dire les conditions du fonctionnement de la signification)" 3 .

$\mathrm{Si}$, comme Saussure l'a proposé, la sémiologie est cette science qui apprend "en quoi consistent les signes" et "quelles lois les régissent", historiquement, on a retenu ce terme pour nommer les théories vouées à la détermination des règles qui gouvernent les différents systèmes de communication. Ainsi a-t-on vu naître, par exemple, une sémiologie du cinéma qui a cherché à définir les codes cinématographiques. En ce qui concerne le théâtre, l'objectif de cette sémiologie est d'étudier la théâtralité comme

2. É. Ertel, “Éléments pour une sémiologie du théâtre», Travail théâtral, n²9, 1977, p. 141.

3. Greimas et Courtès, Sémiotique/Dictionnaire raisonné de la théorie du langage, Paris, Hachette, 1979, p. 249. 
on a étudié la littérarité; la théâtrologie se développant sur le modèle de la narratologie, toutes deux étant des poétiques. Même s'il n'y a pas de langage théâtral proprement dit, cette sémiologie peut tout de même chercher à décrire des codes spécifiquement théâtraux, qu'ils soient dramaturgiques ou spectaculaires.

\section{La sémiologie du texte dramatique}

Ainsi, quand elle s'intéresse au texte dramatique, la sémiologie examine les principes de construction d'une œuvre (exposition, nœud, dénouement, etc.), son découpage (actes, scènes, tableaux); et elle relève les caractéristiques formelles de l'écriture dramatique, qui sont, est-ce utile de le préciser, différentes de celles de l'écriture romanesque, pour ne prendre que cette comparaison. Par exemple, une telle étude démontrerait que le texte dramatique, parce qu'il est écrit pour être joué, est composé de deux niveaux de discours, ayant chacun un énonciateur différent : l'auteur et le personnage. Le premier est le niveau didascalique - ou tout ce qui relève de l'indication scénique, c'est-à-dire toutes les informations que l'auteur juge pertinent de donner à un metteur en scène éventuel pour "guider» le travail d'actualisation de son texte à la scène : noms des personnages et renseignements (âge, sexe, caractéristique physique, etc.), entrées et sorties, changements d'interlocuteurs ou actions quelconques, informations sur l'espace et le temps dramatiques, ou encore des didascalies à fonction dite «mélodique», qui spécifient une modalité d'énonciation en proposant une attitude, une mimique, etc. Une des tâches de la sémiologie du texte dramatique est de proposer une typologie de ces didascalies.

Le deuxième niveau de discours est celui de la parole des personnages. Cette parole peut se présenter sous des formes différentes - le dialogue, le monologue, le chœur et la narration (on connaît l'importance du retour du narra- 
teur sur les scènes de théâtre depuis Brecht). Décrire les particularités langagières de ces discours apparaît immédiatement comme une autre tâche susceptible d'occuper les sémiologues. Ici, il serait intéressant de noter l'apport très enrichissant de la théorie des actes de langage, élaborée principalement par Austin et Searle, qui contribue à montrer comment la parole, particulièrement au théâtre, est ACTION. À titre d'exemple, il n'est pas difficile de mesurer l'impact d'une réplique comme "oui, je te vengerai» sur le déroulement d'une action dramatique; une telle phrase serait, selon le jargon de cette théorie, un énoncé performatif ayant un effet "perlocutoire" notoire : celui de produire l'action, de l'engager dans un sens, celui aussi d'établir des rapports entre des personnages, ce qui est le fondement de tout texte dramatique.

Toujours du côté de l'analyse du texte dramatique, il faudrait mentionner aussi que la sémiologie théâtrale emprunte à la narratologie au moins deux de ses outils d'analyse : la logique du récit et le système actantiel (cf. Propp, Greimas, Brémond, Hamon, Genette). Pour l'examen de textes proposant ce que l'on appelle communément une "histoire", on a en effet souvent recours à ces modèles qui permettent de rendre compte de la logique du développement d'une fable (son découpage révélant les relances de l'action) et de percevoir les enjeux de l'action en dévoilant les rapports de force entre les personnages, vus comme des "actants" (et non comme des "personnes"). II faut faire remarquer également les particularités du traitement de l'espace et du temps au théâtre. Ces considérations peuvent contribuer non seulement à mieux lire certains textes mais également à constituer une typologie des genres dramatiques (tragédie, comédie, drame) et des esthétiques (naturaliste, symboliste, épique, etc.).

On le voit, il y a de la place pour une sémiologie diachronique qui s'intéresserait, dans une perspective historique, au développement des signes textuels du texte 
dramatique; ce qu'elle nous apprend est fort utile pour une réception moins "naïve» des différents spectacles, et facilite l'appréciation de l'originalité des propositions qui nous viennent des praticiens.

\section{La sémiologie de l'acte théâtral}

Cependant, puisque le théâtre est aussi et même fondamentalement spectacle, la sémiologie ne doit pas considérer seulement le texte dramatique. S'imposent plutôt à l'analyse les conventions du spectacle sur lesquelles l'événement théâtral est fondé. Ainsi, la communication théâtrale est-elle régie par des codes spécifiquement théâtraux - alors que le texte l'est par des codes dramaturgiques - , codes dont relèvent, par exemple, des signes comme le lever du rideau ou les trois coups traditionnels qui signifient le début du spectacle, la fonction ludique des éléments de la scène, les entrées et les sorties qui ponctuent les scènes, etc. Bref, ce sont des conventions culturelles instituant un contrat entre les émetteurs et les récepteurs du spectacle.

Tout ce que cette sémiologie des codes - dramaturgiques et théâtraux - nous apprend, d'une façon déductive, de l'examen d'ensembles de pièces, aide à faire mieux comprendre le fonctionnement interne du spectacle théâtral, les principes de son organisation. Toutefois, si l'objectif est de proposer une LECTURE d'un spectacle, même si cette sémiologie est appelée à contribuer à une connaissance des codes qui le régissent, elle ne réussit pas à rendre compte de la complexité de toutes les opérations de signification que propose un spectacle théâtral. Car pour comprendre un spectacle, s'il faut être minimalement conscient de ces conventions, il faut surtout interpréter les signes qui sont proposés. C'est alors qu'on laisse la sémiologie de la communication pour une sémiologie de la signification. 


\section{La sémiotique du texte spectaculaire}

Actuellement, c'est justement cette différence d'objet qui fonde la distinction entre sémiologie et sémiotique. En effet, si l'école européenne, dans le sillage de la linguistique saussurienne, s'est d'abord intéressée à ce phénomène du code théâtral (encore une fois, qu'il concerne le texte dramatique et ses particularités ou la réalité de la représentation), on peut dire que ceux qui ont plutôt opté pour une analyse de la manifestation spectaculaire - la production et la réception d'un spectacle - se situent du côté de la sémiotique qui se veut une science de la signification des signes en contexte, s'inspirant des théories de l'Américain Charles S. Peirce. II ne s'agit plus alors d'une sémiologie du code mais d'une sémiologie du message, pour revenir à la distinction d'Évelyne Ertel. Pour éviter la confusion, et nous écarter de ce modèle saussurien, nous parlerons désormais d'une sémiotique du texte spectaculaire.

On pourrait alors avancer que la sémiotique théâtrale est une critique d'interprétation méthodique, c'est-à-dire une critique qui passe par une description de l'objet à interpréter, élaborée à partir d'une théorie de la signification.

Toute la tentative sémiotique s'inscrit dans l'espace créé par la dénonciation de l'empirisme spontanéiste et intuitif qui régnait jusqu'alors dans la perception des significations. Sera appelé sémiotique tout effort consécutif à cette prise de conscience et visant à repérer, nommer, dénombrer, hiérarchiser d'une façon systématique et objective les unités de signification et leur organisation en ensembles de toutes dimensions ${ }^{4}$.

Ainsi parmi les sciences qui traitent du spectacle, la sémiotique théâtrale s'est-elle taillée la place d'un métadiscours produisant une description de la signification par le biais d'un repérage formel d'unités que constituent les signes

4. A. Hénault, Les Enjeux de la sémiotique, Paris. PUF, 1979, p. 17. 
du spectacle, dans le but d'améliorer la lecture de ce produit culturel spécifique.

Cette approche constitue ce qu'André Helbo a appelé la deuxième phase de la sémiotique theâtrale, quand il a fait le bilan des recherches au Colloque de l'Association internationale pour la sémiologie du spectacle, en 1981 :

On se souviendra que, considérée sous l'angle de son développement, la sémiologie du spectacle a connu deux temps forts :

a) tournée vers le théâtre et inspirée par les sémiologies-sœurs du cinéma ou du récit, elle a tenté de résoudre des problèmes de découpage, d'unité, de migration scénico-textuelle,

b) s'émancipant d'une certaine frustration devant l'aléatoire, la sémiologie du théâtre a par la suite abordé la théorie de la réception, l'événement de la représentation ${ }^{5}$.

Inspirée de la sémiotique américaine qui, depuis Peirce et Morris, considère qu'un exercice sémiotique doit être mené aux trois niveaux de la sémantique, de la syntaxe et de la pragmatique, la sémiotique du spectaculaire décrit le spectacle en fonction de ses relations avec son collectif d'émetteurs, ses récepteurs et ses cadres de références. On voit bien ici le tournant qui fait de la sémiotique une théorie du signe, de la signification et de la réception. Cette prise de position exige une discussion sur les modes d'encodage et de décodage du message théâtral entraînant par le fait même une analyse des rapports entre le signe et l'extrathéâtral (toute la question du référent) et la prise en considération du travail productif du spectateur qui s'engage, selon l'expression d'Umberto Eco, dans un exercice de "coopération textuelle» 6 .

5. A. Helbo, «Perspectives", Degrés, no 29, 1982, p. a-3.

6. Cf. U. Eco, Lector in fabula, Paris, Grasset, 1985. 
Afin d'échapper au "délire interprétatif» et pour répondre à des exigences d'ordre "scientifique", cette approche propose des choix méthodologiques en ce qui concerne, par exemple, le découpage du corpus, une approche fonctionnelle des unités signifiantes et la recherche d'une structure organisatrice de l'œuvre.

II ne s'agit pas de prétendre attribuer quelque sens (dé)fini à une œuvre - on ne cherche pas sa "vérité" mais plutôt de susciter la curiosité de la découverte des nombreuses lectures possibles et surtout de montrer l'intérêt d'une lecture systématique qui expose les traces de la production et suit ses effets. Il faut éviter à la fois le "terrorisme du signifiant" et le "terrorisme du signifié", comme le dit Michel Corvin, et en arriver à articuler les deux questions : "qu'est-ce qui constitue la matière signifiante?» et "qu'est-ce que ça signifie?»?.

La tendance actuelle de la sémiotique théâtrale est donc de mettre l'accent sur la communication théâtrale en tant que "procès global sémantico-pragmatique" (l'expression est de Marco de Marinis). En effet, plusieurs études antérieures, d'ailleurs souvent laissées en plan, qui voulaient définir l'écriture scénique et élaborer un «langage dramatique", comme on l'a vu, semblent avoir oublié le destinataire du message théâtral. Or, il sera impossible dorénavant de négliger les conditions d'énonciation et de perception au théâtre. On est à la recherche des stratégies discursives et des effets de ce discours.

Si la spécificité du théâtre réside dans le fait que la signification s'élabore à partir de signifiants multiples, c'està-dire qu'elle circule dans des matériaux hétérogènes plutôt que d'être le résultat d'une addition pure et simple, il faut axer les recherches sur cette opération de circulation

7. M. Corvin, «À propos de deux spectacles de Robert Wilson - essai de lecture sémiologiquen, Cahiers de la compagnie M. Renaud-J.L. Barrault, n० 7, 1971, p. 91. 
du sens d'une complexité assez étonnante, plutôt que sur la détermination à tout prix d'une unité minimale d'analyse comme le «signe théâtral».

La tâche d'une sémiotique théâtrale aujourd'hui n'est plus seulement, d'ailleurs peut-être plus du tout, d'isoler des signes en vue de la constitution de systèmes sémiotiques séparés mais réside plutôt dans un effort d'intégration du signe dans la structure globale que constitue un spectacle singulier. La relation du signe avec cet ensemble apparaît plus riche d'intérêt que les relations qu'il entretient avec un système spécifique, extérieur, duquel il est tiré. Non seulement l'existence d'un code théâtral unique estelle de plus en plus aléatoire, mais le signe lui-même, en tant qu'unité, ne sera plus la pierre de touche de la sémiotique théâtrale. En effet, les questions relatives à la combinatoire et à l'articulation des signes entre eux apparaîtront bientôt déterminantes.

L'intérêt d'une sémiotique théâtrale est [...] non pas de donner des sens aux signes comme on pourrait le croire (ils en ont toujours un ou plusieurs puisque, si j'ose dire, ils sont là pour cela), ni même repérer les signes les plus usuels avec leur sens (l'observation raffinée des historiens du théâtre y suffit bien), mais au contraire de montrer l'activité théâtrale comme constituant des systèmes de signes qui n'offrent de sens que les uns par rapport aux autres. La tâche d'une sémiotique théâtrale est moins d'isoler les signes que de constituer avec eux des ensembles signifiants et de montrer comment ils s'organisent $^{8}$.

\section{Le texte spectaculaire}

Comme la recherche d'،un modèle de spectacle

8. A. Ubersfeld, L'École du spectateur, Paris, Éditions Sociales, 1982, p. 21. S'efforçant de se dégager du formalisme russe, les chercheurs du Cercle linguistique de Prague avaient, déjà dans les années 1930-1940, pris clairement position sur ce qui devait constituer l'objet principal de l'analyse sémiologique : la fonction du signe; of. L. Matejka, "Postscript : Prague School Semiotics», Semiotics of Art - Prague School Contribution, MIT Press, 1976, p. 272. 
considéré comme modèle de tous les spectacles possibles" lui semble peu féconde, Marco de Marinis proposera une sémiotique du texte spectaculaire. Ses définitions de texte spectaculaire et de structure textuelle du spectacle sont très utiles pour l'analyse de représentations. Elles sont d'abord fondées sur la prémisse que l'objet dont on traite est un texte, c'est-à-dire une unité organisée :

Un texte ne représente pas une simple succession de signes dans l'intervalle de deux limites externes. Une organisation interne est propre au texte, qui le transforme, au niveau syntagmatique, en un tout structurel. C'est pourquoi, pour reconnaître un ensemble de phrases de la langue naturelle comme texte artistique, il convient de se convaincre qu'elles forment une structure de type secondaire au niveau de l'organisation artistique. II convient de noter que le caractère structural et la délimitation d'un texte sont liés?.

Tout spectacle sera considéré comme un texte artistique. Évidemment, le terme de texte s'élargit ici pour désigner une organisation composite, faite d'éléments linguistiques et non linguistiques : le texte spectaculaire.

Nous appellerons textes spectaculaires (abréviation TS) ces unités de manifestation théâtrale que sont les spectacles, pris dans leur aspect de "procès» signifiants complexes, à la fois verbaux et non verbaux ${ }^{10}$.

Cette notion de TS est une notion descriptive qui se rapporte au spectacle en tant qu'objet empirique; est analysée ici une manifestation concrète. Plus précisément, le TS est un macro-texte; parce que la première caractéristique du théâtre est d'être un objet composite au niveau de sa matière d'expression, on doit le considérer comme un

9. Y. Lotman, Structure du texte artistique, Paris, Gallimard, 1973, p. 93.

10. M. De Marinis, "Le spectacle comme texte", Organon 80 - Sémiologie et théâtre, CERTC, 1980, Université de Lyon II, p. 197. 
ensemble regroupant ce qu'on appelle des textes partiels (T.P.) : l'espace, les objets, le jeu. La deuxième caractéristique du TS, et de ses TP, réside dans le fait qu'ils sont constitués de signes régis par plusieurs codes. Ainsi le TS jouit-il d'une double hétérogénéité, une hétérogénéité matérielle et une hétérogénéité codique. Partant de la constatation de cette coprésence, la sémiotique théâtrale traitera de leur interaction.

Marco de Marinis passe d'une notion descriptive à une notion théorique et propose le terme de structure textuelle du spectacle (Str.TS) pour désigner les principes organisationnels du TS. Mais, attention, si le TS est préexistant à l'analyse, il n'en va pas de même pour la Str.TS; elle est une "construction" de l'analyse. C'est cette construction qui confère au TS sa cohérence :

Appelons structure textuelle du spectacle le système résultant de la combinaison hiérarchisée des codes (et sous-codes) du TS. Les deux caractéristiques principales de la Str.TS sont la systématisation et la singularité"1.

La tâche de l'analyse est de constituer cette structure textuelle : elle doit nommer et montrer comment s'articulent les codes qui traversent le spectacle, qu'ils soient spécifiquement théâtraux ou non, sans oublier de mentionner que le spectacle modalise lui-même ces codes (il ne se contente pas de les utiliser, il les transforme). L'intérêt d'une telle analyse se trouve tout entier dans la découverte du code singulier d'un texte spectaculaire. On l'appelle singulier parce qu'il est une organisation originale et cohérente. Ainsi une structure textuelle agit-elle à la façon d'un idiolecte (c'est ainsi qu'Eco appelle un code privé), c'est-à-dire qu'elle joue le rôle d'un principe organisateur qui peut ordonner les différentes manifestations de signification qui se rejoignent pour constituer le sens du spectacle. 


\section{Le rôle de la redondance}

Si un texte spectaculaire véhicule des informations complémentaires et propose un réseau sémantique connotatif cohérent, s'il réussit, ce faisant, à provoquer des émotions et des jugements, bref s'il est efficace, c'est qu'il est bien organisé sémantiquement et structurellement. Mais comment découvrir cette cohérence structurelle, comment mettre à jour les "stratégies discursives" qu'il déploie pour guider ses récepteurs vers une lecture satisfaisante de ce qu'il propose?

Comme dans tout acte de communication, le phénomène de redondance est fondamental pour assurer la cohésion sémantique d'un message. Michel Corvin, dans ses études de représentations théâtrales, examine différents types de redondance et arrive à démontrer que ce phénomène est la base même du code singulier d'un texte spectaculaire ${ }^{12}$. Ces types de redondance sont : la redondance fonctionnelle, expressive et structurale.

La première - d'ailleurs très répandue dans tous les types de messages - en assure la lisibilité. On sait depuis les recherches en théorie de la communication qu'un récepteur ne peut "encaisser" autant d'informations qu'il y a de signes. Les signes doivent se "chevaucher" pour que le message soit compris. Ainsi, au théâtre, les informations sur le temps dramatique, par exemple, pourront être distillées dans autant de signes que des accessoires, des costumes, un certain lexique, etc. Ce type de redondance, fonctionnelle, garantit la cohésion sémantique. II va sans dire que cette redondance est la plus élémentaire.

La redondance expressive, elle, parce qu'elle ne se contente pas de confirmer des informations, disons dénotatives, mais joue plutôt dans la sphère plus floue, et plus

12. M. Corvin, Molière et ses metteurs en scène aujourd'hui - Pour une analyse de la représentation, Presses Universitaires de Lyon, 1985. 
riche, de la connotation, apparaît plus intéressante à examiner. On peut observer, par exemple, dans un texte spectaculaire, la réutilisation d'un même signe à différents moments, cette redondance - qui n'est jamais simple répétition puisque ce signe, chaque fois, est dans un rapport contextuel différent - contribue à tisser un réseau de sens qui multiplie les effets, nuance les propos, crée, d'une manière esthétique, une isotopie sémantique fondamentale, voire des symboles. À cette redondance d'un même signe que l'on peut suivre tout le long du T.S., on peut ajouter la redondance d'un "sens", d'une même "idée", pouvant être véhiculée par plusieurs signes matériellement différents. La "violence", par exemple, peut être exprimée par des paroles, des gestes, elle peut même être suggérée par un décor. Il est assez facile de voir l'intérêt qu'un observateur aurait de chercher à dépister ce type de récurrence connotative avant de proposer une lecture d'un texte spectaculaire.

Enfin, la redondance structurale va encore plus loin, si l'on peut dire, que la redondance expressive, dans le sens qu'elle est une systématisation d'une redondance expressive. Si un procédé discursif, une caractéristique stylistique, un trope, se remarque dans plus d'un texte partiel, on pourrait bien y voir une des règles de l'organisation de l'ensemble. Le recours systématique à la parodie, par exemple, dans les programmes gestuels des personnages et dans l'utilisation du discours pourrait constituer une telle règle pour la construction de la structure textuelle de ce spectacle. Ainsi, le phénomène de la redondance est-il un indice sûr pour qui cherche les principes de structuration d'une œuvre.

\section{Les textes partiels}

Mais avant de s'engager dans un tel exercice, il est certainement utile de considérer le «travail» des signes en les regroupant dans le simple but de faciliter leur observa- 
tion. La première étape de l'analyse consisterait donc à découper le texte spectaculaire en textes partiels, ensuite à procéder à la constitution des structures textuelles partielles et globales. Mais d'abord, quels sont les critères du découpage dans la matérialité du spectacle?

Anne Ubersfeld a proposé, dans son École du spectateur, d'étudier trois systèmes de signes à partir de ce qu'elle appelle la reconstruction "de la représentation comme un monde possible" par le spectateur :

1) l'espace non seulement dans ses coordonnées ou dans le lieu qu'il représente, mais dans le mode de relation qu'il suppose entre les protagonistes, et aussi entre la scène et le public;

2) les objets, compris comme référence au monde, mais aussi comme éléments ludiques pour le comédien et envisagés à la fois dans leur matérialité (origine, matériau, temporalité, usure, etc.) et dans leur fonctionnement rhétorique, comme métaphores, métonymies, symboles;

3) le comédien en tant que producteur d'un discours verbalgestuel, mais aussi dans ses rapports avec ses destinataires, protagonistes et public...13

Comment en est-elle venue à ces trois systèmes? Elle avoue avoir procédé à un découpage fondé sur la perception du spectateur, non pas tellement comme Tadeuz Kowzan qui distinguait signes visuels et signes auditifs, mais plutôt d'après ce qu'elle appelle des "ensembles textuels déjà constitués", c'est-à-dire perceptibles isolément. Ce qui différencie grandement cette position de celle de Kowzan, c'est que ces ensembles ont déjà subi (par le travail du producteur) une certaine organisation des signes qui les constituent en ensembles. Ainsi, "l'ensemble" comédien, producteur d'un discours verbal et gestuel, regroupe-t-il des signifiants de substances différentes.

13. A. Ubersfeld, op. cit., p. 322. 
La segmentation, bien sûr, n'est que provisoire et d'ordre utilitaire. Par souci d'efficacité, on parle de trois systèmes : espace, objets et jeu; on précise que leur distinction repose sur le fait que le spectateur les perçoit d'une façon discontinue. Le choix de ces trois textes partiels réussit pourtant à définir la spécificité du mode d'expression qu'est l'art théâtral. En effet, on y retrouve les éléments essentiels à l'activité théâtrale qui est la rencontre, en un temps et un espace définis, d'un groupe de récepteurs et d'un groupe d'émetteurs s'activant à créer un univers fictif. Si l'essence du théâtre, comme le disaient déjà Honzl et les sémioticiens du Cercle linguistique de Prague, réside dans l'action dramatique, les trois systèmes d'Ubersfeld le confirment : c'est dans un système "englobant", soit l'espace, matériellement organisé pour proposer un univers fictif, que l' «action» du corps (gestes et paroles) est perceptible. II est d'autant plus important de considérer l'action comme le moteur de l'événement théâtral que cette action est le lieu même de la rencontre des signes; cela évite de considérer la signification comme une simple juxtaposition de sens et invite à une lecture globale. Examiner d'abord d'une manière séparée ces systèmes n'implique pas qu'on les considère comme des systèmes clos; au contraire, cela permet de mieux voir les interactions et devrait faciliter l'étude du "courant unique» qui traverse le texte spectaculaire.

L'analyse de ces T.P. doit se faire à plusieurs niveaux. Ils peuvent être examinés séparément, puis en relation les uns avec les autres. L'analyse doit respecter les particularités de la production théâtrale qui présente simultanément au spectateur plusieurs signes de systèmes différents et qui s'étale dans le temps. Ce qui implique qu'à l'intérieur de chaque système, on fasse une analyse sémique des signes qui le composent et qu'on s'intéresse aux relations qu'ils entretiennent entre eux. Par exemple, un relevé systématique des signes d'un texte partiel, exercice préalable 
à toute analyse, permettra de construire des paradigmes utiles pour fonder une lecture isotopique de l'ensemble.

Très tôt, par contre, l'analyse doit devenir syntagmatique en opérant des coupes "photographiques" d' "événements scéniques", comme les appelle Anne Ubersfeld, qui permettent de voir «travailler» ensemble des signes de systèmes différents. II est révélateur que Michel Corvin, tout comme Anne Ubersfeld, propose l'emploi du mot syntagme pour désigner l'unité signifiante à étudier plutôt que le mot signe. Ce dernier est trop relié à une opération de découpage systémique en unités de plus en plus petites, lequel découpage nuit finalement plus qu'il n'aide à la compréhension de l'ensemble.

Les syntagmes sont des unités plurisystémiques où s'observe très bien l'action du code singulier. Leur analyse permet de dégager certaines règles de ce code.

\section{L'espace}

Étudier l'espace au théâtre exige d'abord qu'on en distingue quatre types : le lieu théâtral, la bâtisse (un théâtre ou non) qui reçoit le spectacle et dont l'architecture influence les conditions d'énonciation; l'espace scénographique qui est l'organisation spatiale de l'intérieur de la salle où a lieu le spectacle et qui se divise en deux zones, l'une pour le jeu, l'autre pour le public - cette organisation, qui n'est fixe que dans les théâtres traditionnels avec scène à l'italienne, instaure les rapports entre le spectacle et ses récepteurs, et crée chaque fois des conditions particulières d'émission et de réception du discours; l'espace scénique, c'est-à-dire l'espace créé par la rencontre dynamique des signes scéniques - décor, accessoires, mouvements et déplacements des personnages - constituant le lieu même de la représentation de l'espace dramatique qui est, lui, l'espace fictif suggéré par le texte dramatique (spécifions que cet espace dramatique est souvent d'ordre locatif mais qu'il peut aussi être "psychique"). 


\section{Les objets}

Le deuxième texte partiel, constitué des "objets» dans le texte spectaculaire, est bien vaste, car il faut entendre par "objet» tout ce qui sert au jeu : le décor comme tel, les accessoires et les costumes, mais aussi l'éclairage ou encore le bruitage. Tous ces signes remplissent de nombreuses fonctions qui vont de la fonction purement esthétique à la fonction proprement signifiante, ou référentielle, en passant par des fonctions spatiales, quand ils déterminent des espaces de jeu, ludiques, quand ils servent ostensiblement au jeu (une balançoire, par exemple) ou encore métalinguistiques quand ils dénotent par leur nature même leur appartenance au monde théâtral (le simple exemple du masque suffira à donner une idée de cette fonction).

L'objet au théâtre, à la fois présence et signe, constitue un terrain privilégié pour observer les modes de signification du signe théâtral (métaphore, métonymie et autres tropes), et pour remarquer l'extraordinaire puissance de référence et la mobilité du signe théâtral.

\section{Le jeu}

C'est lors de l'examen du troisième texte partiel, les comédiens en jeu, que l'on peut le plus efficacement mettre l'accent sur les rapports d'interprétance entre les signes. En effet, la corrélation entre tous les éléments signifiants est d'autant mieux observable que l'on étudie alors un système dynamique, c'est-à-dire en mouvement, en transformation, en progression. Le jeu est le fondement même de l'activité théâtrale, le lieu d'émergence de la signification. Son étude permet de rallier les observations faites à propos des unités d'ordre spatial et des divers objets à l'examen des discours, verbaux et gestuels, produits par les comédiens.

On analyse alors les figures scéniques que les comé- 
diens proposent des personnages dramatiques, figures qui se construisent à partir de programmes gestuels censés leur donner cohérence, lisibilité et, surtout, contribuant à établir leurs fonctions actantielles. Les déplacements, les gestes, la mimique, le port de tête, tout participe à ces figures scéniques. Les programmes gestuels, évidemment, dépendent de l'esthétique globale du texte spectaculaire, comme ils y contribuent. Ainsi le programme gestuel d'un personnage naturaliste restera-t-il toujours dans le registre du vraisemblable. II n'en va pas de même, toutefois, d'une gestuelle stylisée ou du gestus brechtien qui veut souligner l'empreinte du social sur l'individu.

\section{L'interprétant}

Chose indispensable, il faut articuler cette approche fonctionnelle des signes théâtraux et une théorie de la signification. Les recherches de Charles S. Peirce, particulièrement en ce qui concerne son concept d'interprétant (on aura remarqué déjà l'usage du mot «interprétance» à quelques reprises), sont en train de se révéler fondamentales pour l'analyse du théâtre. L'interprétant, comme lieu de circulation de la signification, pourrait bien constituer le concept charnière entre une théorie du signe, ou de la semiosis, et une analyse pratique, une description de la structure signifiante d'un objet culturel particulier.

En sémiotique théâtrale, on a emprunté, un peu hâtivement peut-être, au sémioticien américain ses notions d'indice et d'icône (Pavis, Demarcy, Ertel, Ubersfeld en ont parlé). Toutefois, il faudrait aujourd'hui se démarquer de cette voie en insistant plutôt sur l'importance à accorder à la notion d'interprétant, liée à la production du sens ${ }^{14}$. On

14. Au bénéfice des lecteurs, peut-être est-il requis de rappeler qu'un signe, selon Peirce, est une structure à trois faces qui met en relation un premier, un representamen (ce qui tient lieu de signe), un deuxième, son objet (ce à quoi renvoie le signe "dans la réalité") et un troisième, son interprétant (soit ce qui "émerge" quand le signe est produit et saisi). 
a négligé de respecter tous les aspects de la théorie peircienne; une de ses principales qualités est de présenter le signe comme une entité à trois dimensions (l'icône et l'indice ne s'expliquent que par des rapports entre le représentamen (le signe lui-même) et l'objet sans l'intervention de l'interprétant). La sémiotique théâtrale doit prendre en considération tous les niveaux de rapports entre ces dimensions pour vraiment démontrer que la semiosis théâtrale est un phénomène circulaire, en contexte. Précisons qu'on perçoit un signe, mais qu'on en saisit les effets par le biais de l'interprétant.

L'interprétant, c'est la somme des sens qui se raccrochent à un signe dans le contexte précis où on le trouve : un sens que lui accordent un code fixe s'il y en a un, et toutes les associations mentales, les pensées qu'il déclenche dans l'esprit du récepteur; ce peut être aussi, moins précisément, les effets que le signe produit chez le récepteur : une réaction physiologique (dégoût, euphorie, pleurs...), une réaction émotive ou affective, un sentiment (par exemple, quand on écoute de la musique), un souvenir (tout ce qui vient à l'esprit d'un parent quand il voit un enfant sur la scène), un rappel d'un détail d'un produit culturel perçu antérieurement (un livre, un film, une publicité, un spectacle); ce peut être même une action (quand je suis au coin de la rue et que je démarre au feu vert, mon action est un interprétant du signe $)^{15}$.

Cette théorie du signe permet, d'une part, d'analyser le travail de "migration" des signes linguistiques du texte dramatique aux signes scéniques du texte spectaculaire, et, d'autre part, de produire une lecture de tout acte de signification; elle est donc utile à la fois aux praticiens qui proposent, littéralement, leur interprétation d'un texte d'auteur (comme on le dit d'une interprétation musicale) et aux récepteurs qui, à leur tour, sont engagés dans un exercice

15. L. Vigeant, La Lecture du spectacle théâtral, Laval, Mondia, 1989, p. 13. 
de lecture interprétative du résultat de ce travail alors qu'ils lui construisent un sens.

Ainsi, si la sémiologie permet un inventaire des différents codes dramaturgiques et théâtraux, et leur histoire, la sémiotique, elle, parce qu'elle se présente comme une pragmatique, contribue à éclaircir les phénomènes complexes de signification, de tous ordres, que l'on rencontre au théâtre. À ce titre, elle mérite sa place dans les sciences humaines censées nous aider à comprendre les multiples discours qui définissent notre société.

Louise Vigeant Collège Édouard-Montpetit 


\section{Bibliographie à consulter}

Aristote, La Poétique.

Austin, J.L. [1970] : Quand dire, c'est faire, Paris, Seuil.

Barthes, R. [1985] : L'Aventure sémiologique, Paris, Seuil.

Brémond, C. [1973] : La Logique du récit, Paris, Seuil.

Corvin, M. [1985] : Molière et ses metteurs en scène aujourd'hui - Pour une analyse de la représentation, Lyon, PUL.

Deledalle, G. [1979] : Théorie et pratique du signe, Paris, Payot.

De Marinis, M. [1980] : “Le spectacle comme texte», Organon - Sémiologie et théâtre, CERTC, Université de Lyon II.

Eco, U. [1972] : La Structure absente: introduction à la recherche sémiotique, Paris, Mercure de France.

[1965] : L'CEuvre ouverte, Paris, Seuil.

[1985] : Lector in Fabula, Paris, Grasset.

[1988] : Le Signe. Histoire et analyse d'un concept, Bruxelles, Labor.

Elam, K. [1980] : The Semiotics of Theatre and Drama, New York, Methuen.

Greimas, A. [1966] : Sémantique structurale, Paris, Larousse.

Helbo, A. [1975] : Sémiologie de la représentation, Bruxelles, Complexe.

[1983]: Les Mots et les gestes. Essai sur le théâtre, Bruxelles, Complexe. 
Helbo, Johansen, Pavis, Ubersfeld, [1987] : Théâtre, Modes d'approche, Bruxelles, Labor.

Kerbratt-Orecchioni, C. [1977] : La Connotation, Lyon, PUL.

Kowzan, T. [1975] : Littérature et spectacle, Varsovie, PWN-Éditions scientifiques de Pologne.

Lotman, Y. [1973] : Structure du texte artistique, Paris, Gallimard.

Monod, R. [1977] : Les Textes de théâtre, Lyon, CEDIC.

Pavis, P. [1982] : Voix et images de la scène, Lille, Presses Universitaires de Lille.

[1980] : Dictionnaire du théâtre, Paris, Éditions Sociales.

Peirce, C.S. [1978] : Écrits sur le signe, Paris, Seuil.

Propp, V. [1965, 1970] : Morphologie du conte, Paris, Seuil, Coll. Points.

Purkhardt, B. [1986] : "Les trois coups de la communication théâtrale", La petite revue de Philosophie, vol. 7, no 2.

Ubersfeld, A. [1977] : Lire le théâtre, Paris, Éditions Sociales. ciales.

[1981] : L'École du spectateur, Paris, Éditions So-

Vigeant, L. [1989] : La lecture du spectacle théâtral, Laval, Mondia.

Villeneuve, R. [1982, 1983] : “Un discours théâtral?", Cahiers de theâtre JEU, no 24, p. 57-67. 